\title{
Editorial
}

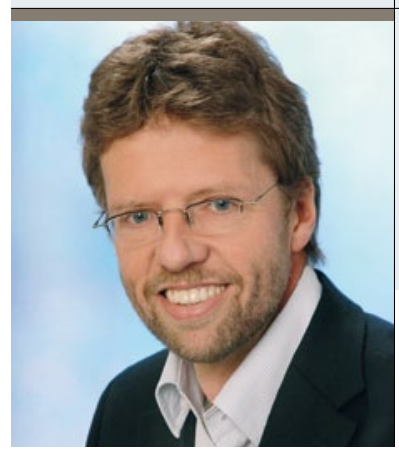

„In Deutschland warten noch ungefähr 180.000 Menschen

auf neue Therapien, um ihre Epilepsie endlich in den Griff

zu bekommen."

\section{Bleiben die Patienten auf der Strecke?}

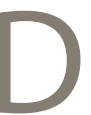
as neue Antiepileptikum Retigabin wird nach kurzer Zeit vom deutschen Markt genommen. Wie konnte es dazu kommen? Der Gemeinsame Bundesausschuss (G-BA) hat am 3. Mai 2012 eine abschließende frühe Nutzenbewertung zum neuen Antiepileptikum Retigabin herausgegeben, das seit Herbst 2011 auf dem Markt verfügbar ist. Der G-BA kam nach der Analyse umfangreicher Dossiers und einer Anhörung aller Beteiligten zu dem Schluss, dass das Medikament in der Add-on-Therapie fokaler Epilepsien keinen Zusatznutzen gegenüber der „zweckmäßigen Vergleichstherapie " mit Lamotrigin und Topiramat besitzt. Der Hersteller, Glaxo-SmithKline (GSK), hatte in seinem Dossier Lacosamid als Vergleichstherapie gewählt, weil dieses relativ neue Medikament ebenfalls bei Patienten eingesetzt wird, bei denen andere Therapien versagt haben. Der G-BA hielt dagegen Lamotrigin und Topiramat als zweckmäßige Vergleichstherapie für richtig, obwohl beide Wirkstoffe vor allem in der Monotherapie beziehungsweise der frühen Kombinationstherapie zum Einsatz kommen. Studiendaten, welche GSK zum Vergleich von Retigabin mit Lamotrigin und Topiramat nachreichte, blieben in der aktuellen Bewertung des G-BA unberücksichtigt. Der Hersteller kann erst nach zwölf Monaten ein neues Dossier einreichen.

\section{Vom deutschen Markt genommen}

In der Folge hat GSK von der „Opt out“-Möglichkeit Gebrauch gemacht, keine Preisverhandlungen aufund das Medikament vom deutschen Markt genommen. Bis zu einer erneuten Nutzenbewertung wird das Medikament daher ab sofort in Deutschland nicht mehr im Verkehr und auch nicht mehr allgemein erstattungsfähig sein. Retigabin muss nun über die internationale Apotheke bestellt werden und die Kassen müssen vorab zur Kostenübernahme angefragt werden. AOK und TK haben unlängst signalisiert, dies bei bereits auf Retigabin eingestellten Patienten unbürokratisch zu tun.

\section{Was steckt dahinter?}

Das Arzneimittelmarktneuordnungsgesetz (AMNOG) versucht, die Kosten im Gesundheitssystem zu regeln. Der Bundesminister für Gesundheit Daniel Bahr verkündet auf der Webseite des Ministeriums: „Damit die Beiträge zur Krankenversicherung bezahlbar bleiben, dürfen die Ausgaben für Medikamente nicht unbegrenzt steigen. Das Arzneimittelmarktneuordnungsgesetz schafft die schwierige Balance zwischen Innovation und Bezahlbarkeit bei Arzneimitteln“. Vor dem Hintergrund, dass die Arzneimittelkosten höher ausfallen als die Arzthonorare, ist dieser Ansatz an sich sehr zu begrüßen. Im Grundprinzip kann ein Hersteller, der für eine neues Arzneimittel einen Zusatznutzen nachweisen kann, einen höheren Preis verhandeln als für vergleichbare Produkte. Können die Hersteller einen solchen Zusatznutzen nicht nachweisen, empfiehlt der G-BA einen Preis nur in Höhe der Vergleichssubstanzen zu gewähren. In aller Regel sind dies generische und damit meist preisgünstige Pharmaka. Ein solcher Prozess dauert bis zu einem Jahr und beinhaltet auch die Beratung der pharmazeutischen Industrie beim Institut für Qualität und Wirtschaftlichkeit im Gesundheitswesen (IQWIG). Der Hersteller erfährt darin die zweckmäßige Vergleichstherapie und die Anforderungen für die Erstellung eines umfangreichen Dossiers zum Produkt und zum Zusatznutzen. Für die Planung und Durchführung von Zulassungsstudien werden diese Informationen jedoch immer zu spät kommen, zumal in diesen Studien zunächst stets gegen Placebo geprüft wird.

Hat das IQWIG seinen Bericht erstellt, bleiben dem Hersteller drei Wochen für eine Stellungnahme, anschließend erfolgt eine mündliche Anhörung vor dem G-BA, zu der unter anderem auch die Fachgesell- 
Trotz zahlreicher verfügbarer Antiepileptika kann noch immer nicht allen Patienten medikamentös geholfen werden. Neue Wirkstoffe werden daher weiterhin dringend gesucht. Steht die frühe Nutzenbewertung nach AMNOG dem im Weg?

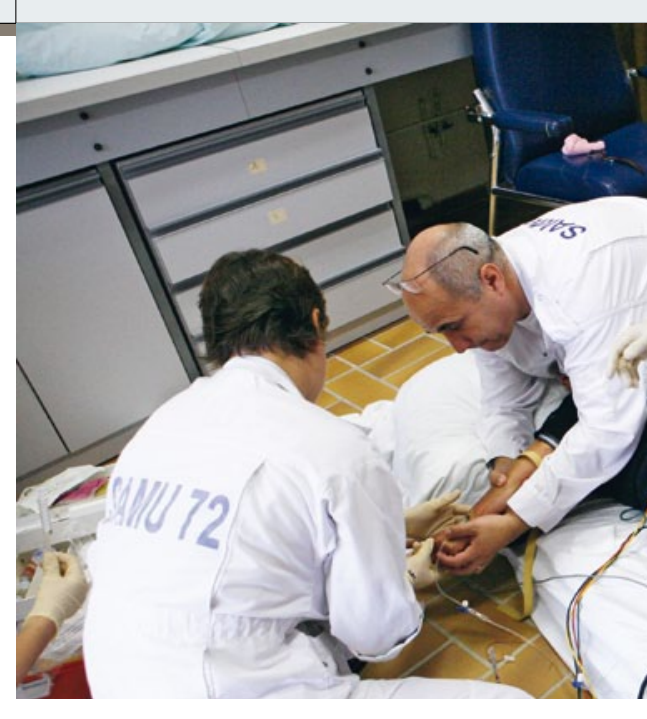
$\left(\frac{10}{10}\right.$

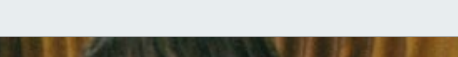

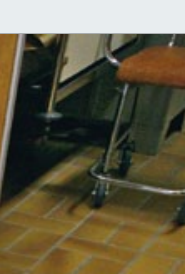

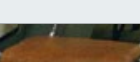

1
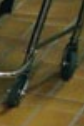

schaften eingeladen werden. Der Beschluss des G-BA, etwa sechs Wochen nach der Anhörung, hat bindenden und juristisch nicht angehbaren Charakter. Im Falle von Retigabin lagen die Einschätzungen, welche Vergleichssubstanz heranzuziehen sei, deutlich auseinander. Die Wahl ist insofern wichtig, weil bei fehlendem oder nicht belegtem Zusatznutzen das neue Medikament die Kosten der Vergleichssubstanz nicht überschreiten darf. Im Falle von Retigabin hätte die Erhaltungsdosis bei Menschen mit fokaler Epilepsie um den Faktor sechs bis zehn günstiger sein müssen als derzeit.

\section{Andernorts pragmatischere Lösungen}

Prinzipiell ist es richtig, dass das Ministerium über eine Neuregelung versucht, die Kosten von Arzneimittel zu drosseln, um die Kosten insgesamt nicht ansteigen zu lassen. Problematisch erscheint, dass bereits viele Patienten mit der jetzt bewerteten Substanz behandelt werden, für die eine Rücknahme des Medikamentes ethisch nicht zumutbar ist. Im Indikationsbereich von Retigabin, therapierefraktären fokalen Epilepsien, ist eine schnelle Marktregulierung dergestalt zu erwarten, dass nur die Patienten darauf eingestellt bleiben, die wirklich davon profitieren. Das sind hierzulande etwa 1.000 Menschen. Klüger wäre es gewesen, die Zulassung stärker einzuschränken und klarer zu definieren, wie es in Schottland und anderen europäischen Ländern der Fall ist. Retigabin kann laut Zulassung bei allen fokalen Epilepsien eingesetzt werden, sofern noch Anfälle bestehen, also bei circa 85.000 bis 150.000 Patienten. Eine genauere Definition, wann und von wem die Substanz wirklich verschrieben werden kann, wäre sinnvoll. Denkbar wäre eine Zulassung erst nach zum Beispiel mindestens fünf erfolglosen, dokumentierten Addon-Vorbehandlungen. Auch die ausschließliche Verordnung in Schwerpunktpraxen für Epilepsie sowie Epilepsieambulanzen würde dem Problem eher gerecht werden.

Auch erscheint es wenig sinnvoll, dass ein so wichtiges und professionell arbeitendes Institut wie das IQWIG in diesem Verfahren keinen der in Deutschland angesehenen Epilepsieexperten als Gutachter akzeptiert hat, mit der Begründung, dass diese Experten auch von der Industrie zu Vorträgen und Beratungs-Boards eingeladen werden und somit potenziell befangen seien. Die Deutsche Gesellschaft für Epileptologie sucht nun das Gespräch mit dem G-BA und Vertretern des IQWIG, damit künftige Entscheidungen nicht zu Ungunsten der Patienten ausfallen. Immerhin warten in Deutschland noch ungefähr 180.000 Menschen auf neue Therapien, um ihre Epilepsie endlich in den Griff zu bekommen. So erwartet der neue antiepileptisch wirksame Wirkstoff Perampanel im Herbst die Beurteilung des G-BA. Eine ähnliche Entscheidung wie bei Retigabin würde die Entwicklung neuer Substanzen in Deutschland völlig zum Erliegen bringen. Es bleibt fraglich, ob es ohne neue innovative Medikamente wirklich zu einer Kostenersparnis kommt. Und schließlich: Ein hocheffektives und sehr gutes Antiepileptikum wie Levetiracetam wäre 2000 kaum auf dem deutschen Markt verblieben, wäre es vom G-BA beurteilt worden. Eine „Head-to-Head“-Studie mit Carbamazepin zeigte nämlich Jahre nach der Zulassung, dass das alte Antiepileptikum Carbamazepin bei neu diagnostizierten Patienten und in Monotherapie keineswegs schlechter war als das neue Levetiracetam [Brodie et al. Neurology 2007; 68: $402-8]$. Aus solchen Vergleichen kann man eigentlich nur schließen, dass das neue Medikament (ebenfalls) etwas taugt. Die differenzierenden Merkmale von Levetiracetam und Carbamazepin haben sich auch erst durch weitere Studien und in der praktischen Anwendung herauskristallisiert.

Für die circa 1.000 auf Retigabin eingestellten Patienten wird es nach dem bisherigen Stand vonseiten der großen Krankenkassen keine Probleme geben, aber einige kleine Kassen erkennen die Problematik nicht und agieren bürokratisch. Eine gewünschte Neueinstellung dürfte allerdings Papierkram mit Einzelanträgen an die Kasse von Arzt und Patient zur Folge haben.

Mit freundlichen kollegialen Grüßen, Ihr

Dr. Thomas Mayer

Kleinwachau, Sächsisches Epilepsiezentrum Radeberg

E-Mail: t.mayer@kleinwachau.de

www.bmg.bund.de/krankenversicherung/arzneimittelversorgung/arzneimittelmarktneuordnungsgesetz-amnog.html www.g-ba.de/informationen/beschluesse/1480/ 